Pré-Publicações do Departamento de Matemática

Universidade de Coimbra

Preprint Number 14-25

\title{
ORTHOGONAL POLYNOMIAL INTERPRETATION OF $\Delta$-TODA EQUATIONS
}

\author{
I. AREA, A. BRANQUINHO, A. FOULQUIÉ MORENO AND E. GODOY
}

\begin{abstract}
The correspondence between dynamics of $\Delta$-Toda equations for the coefficients of the Jacobi operator and its resolvent function is established. A method to solve inverse problem - integration of $\Delta$-Toda equations - based on Padé approximates and continued fractions for the resolvent function is proposed. The main ingredient are orthogonal polynomials which satisfy an Appell condition, with respect to the forward difference operator $\Delta$. Two examples related with Jacobi and Laguerre orthogonal polynomials and $\Delta$-Toda equations are given.
\end{abstract}

KEYwords: Orthogonal polynomials, Difference operators, Operator theory, Toda lattices.

AMS SubJect Classification (2010): 33C45, 37K10, 65Q10.

\section{Introduction}

Many physical problems are modeled by nonlinear partial differential equations for which, unfortunately, the Fourier transform method fails to solve the problem. There was no unified method by which classes of nonlinear partial differential equations could be solved, and the solutions were often obtained by rather ad hoc methods. A significant result was posed by Gardner, Greene, Kruskal and Miura in [6, 7] of a method for the exact solution of the initial-value problem for the KdV equation [13]

$$
u_{t}+6 u u_{x}+u_{x x x}=0,
$$

for initial values which decay sufficiently rapidly, through a series of linear equation, which is now referred to as the Inverse Scattering Transform [2].

Received June 27, 2014.

The work of IA and EG has been partially supported by the Ministerio de Economía y Competitividad of Spain under grant MTM2012-38794-C02-01, co-financed by the European Community fund FEDER. AB acknowledges Centro de Matemática da Universidade de Coimbra (CMUC), funded by the European Regional Development Fund through the program COMPETE and by the Portuguese Government through the FCT - Fundação para a Ciência e a Tecnologia under the project PEst-C/MAT/UI0324/2011. AFM acknowledges Center for Research and Development in Mathematics and Applications (University of Aveiro), funded by the European Regional Development Fund through the program COMPETE and by the Portuguese Government through the FCT-Fundação para a Ciência e a Tecnologia under the project PEst-C/MAT/UI4106/2011 with COMPETE number FCOMP-01-0124-FEDER-022690. 
P. D. Lax in [14] put the inverse scattering method for solving the KdV equation into a more general framework which subsequently paved the way to generalizations of the technique as a method for solving other partial differential equations. He considered two time-dependent operators $\mathcal{L}$ and $\mathcal{M}$, where $\mathcal{L}$ is the operator of the spectral problem and $\mathcal{M}$ is the operator governing the associated time evolution of the eigenfunctions $\mathcal{L} v=\lambda v, \frac{d v(t)}{d t}=\mathcal{M} v$; and hence we get $\frac{d \mathcal{L}}{d t}=\mathcal{L} \mathcal{M}-\mathcal{M} \mathcal{L}$ if, and only if, $\frac{d \lambda(t)}{d t}=0$. If a nonlinear partial differential equation arises as the compatibility condition of two such operators $\mathcal{L}$ and $\mathcal{M}$, then the last equation is called the Lax representation of the partial differential equation and $\mathcal{L}$ and $\mathcal{M}$ is the Lax pair.

Orthogonal polynomials [21,22] have profound connections with several other areas of classical analysis: special functions, moment problems, Hankel and Toeplitz determinants, spectral theory of Jacobi matrices, and random matrices. The more recent connection is to the integrable systems (i.e. systems that are solvable as the KdV equations). Starting in the early 1990s, the connection of the orthogonal polynomials to the integrable systems has been increasingly appreciated and used by the orthogonal polynomial community. Many long-standing problems in the area of orthogonal polynomials have been solved using this connection and several new exciting sides of this connection have been discovered. A very recent one is concerned with the relation between orthogonal polynomials and discrete Painlevé equations [10] using the Riemann-Hilbert formalism for orthogonal polynomials [11].

On the other hand, the Toda lattice [27] appears in several contexts. Algebraic aspects related to integrability can be found in [24]; uses of symplectic geometry to the analysis of the original Toda system in [16]; a relativistic mutation is described in [25]. We do not intend to give a full list of applications but to show the wide range of them.

As it has been discovered by Moser [19, 20], the dynamic of the solutions of the Toda equations

$$
a_{-1} \equiv 0, a_{0} \equiv 1,\left\{\begin{array}{l}
\frac{d a_{n}(t)}{d t}=a_{n}(t)\left(b_{n-1}(t)-b_{n}(t)\right), \\
\frac{d b_{n}(t)}{d t}=a_{n}(t)-a_{n+1}(t),
\end{array} \quad n=0,1, \ldots\right.
$$

with bounded initial data

$$
b_{n}(0) \in \mathbb{R}, \quad a_{n}(0)>0,
$$


corresponds to the simple evolution of the spectral measure,

$$
d \mu(x ; t)=\frac{\exp (-x t) d \mu(x, 0)}{\int \exp (-x t) d \mu(x, 0)},
$$

of a self-adjoint operator, $J(t)$, defined in the standard basis of $\ell_{2}(0, \infty)$, $e_{k}=(0, \ldots, 0,1,0, \ldots)^{T}, \quad k=0,1, \ldots$, by a Jacobi matrix

$$
J(t)=\left(J_{i, j}(t)\right)=\left(\begin{array}{ccccc}
b_{0}(t) & 1 & 0 & & \\
a_{1}(t) & b_{1}(t) & 1 & 0 & \\
0 & a_{2}(t) & b_{2}(t) & 1 & 0 \\
& & \ddots & \ddots & \ddots
\end{array}\right) .
$$

In the case of arbitrary bounded complex initial data, the operator $J(t)$ is no longer self-adjoint and therefore the notion of real valued spectral measure looses sense. However, it was possible to establish a simple evolution of the resolvent function, or Stieltjes function [8],

$$
S(z ; t)=e_{0}^{T} R_{z}(t) e_{0},
$$

for the resolvent operator, $R_{z}(t)=[J(t)-z \mathcal{I}]^{-1}$, associated with the operator $J(t)$ (cf. [3]).

Here we highlight some properties of the resolvent function and of the Padé aproximants of resolvent function (5) which can be taken as a basement for the solution of the spectral problem. In this direction, $S(z ; t)$ defined in (5) satisfies

$$
\begin{aligned}
S(z ; t) & =e_{0}^{T} R_{z}(t) e_{0}=e_{0}^{T}(J(t)-z \mathcal{I})^{-1} e_{0}=e_{0}^{T} \sum_{n=0}^{\infty} \frac{J(t)^{n}}{z^{n+1}} e_{0} \\
& =\sum_{n=0}^{\infty} \frac{J_{1,1}^{n}(t)}{z^{n+1}}=\sum_{n=0}^{\infty} \frac{u_{n}(t)}{z^{n+1}}=\left\langle u(t), \frac{1}{z-x}\right\rangle,
\end{aligned}
$$

where $u(t)$ is the linear functional associated to the Jacobi matrix $J(t)$ [18], we have used [4]

$$
\left\langle u(t), x^{n}\right\rangle=J_{1,1}^{n}(t), \quad n \in \mathbb{N},
$$

and the sequence of moments $u_{n}(t)=\left\langle u(t), x^{n}\right\rangle, \quad n \in \mathbb{N}$, exists.

By definition, the diagonal Padé aproximants of index $n, \Pi_{n}$, for the power series

$$
S(z ; t)=\sum_{n=0}^{\infty} \frac{u_{n}(t)}{z^{n+1}}
$$


is a rational function

$$
\Pi_{n}(z)=\frac{P_{n}(z)}{Q_{n}(z)}, \quad \text { with } \operatorname{deg} P_{n} \leq n \text { and } \operatorname{deg} Q_{n} \leq n,
$$

such that

$$
Q_{n}(z) S(z ; t)-P_{n}(z)=\frac{c}{z^{n+1}}+\cdots
$$

An important property of the Padé aproximants for the resolvent function (5) of the operator (4) is that their numerators and denominators satisfy a three term recurrence relation,

$$
w_{n+1}(z)=\left(z-b_{n}\right) w_{n}(z)-a_{n} w_{n-1}(z), \quad n \in \mathbb{N},
$$

with initial conditions $Q_{0}=1, Q_{-1}=0$ and $P_{0}=0, P_{-1}=1$, where $a_{n}$ and $b_{n}$ are the coefficients of the matrix $J(t)$ in (4). The sequence of monic polynomials $Q_{n}$ are orthogonal with respect to the linear functional $u(t)$. In these conditions we say that $u(t)$ is the regular linear functional associated with the sequence $\left\{Q_{n}\right\}$, assuming that $\operatorname{det} H_{n}(t) \neq 0$, where for $n \in \mathbb{N}$, $H_{n}(t)=\left[u_{i+j}(t)\right]_{i, j=0}^{n}$, are Hankel matrices (see [26]).

Furthermore, if det $H_{n}(t)>0, n \in \mathbb{N}$, then $u(t)$ has an integral representation in terms of a positive Borel measure, $\varrho(x ; t)$, supported on an infinite point set, $I$, of the real line,

$$
\left\langle u(t), x^{n}\right\rangle=\int_{I} x^{n} \varrho(x ; t) d x, \quad n \in \mathbb{N},
$$

and the orthogonality condition reads as

$$
\left\langle u(t), P_{n}(x) P_{m}(x)\right\rangle=\int_{I} P_{n}(x) P_{m}(x) \varrho(x ; t) d x=h_{n} \delta_{n, m}, \quad h_{n}>0, n, m \in \mathbb{N} .
$$

This property is equivalent to the fact that the diagonal Padé approximants are the convergents of the continued fraction expansion for the power series $(7)$, i.e.

$$
S(z ; t)=\frac{1}{z-b_{0}-\frac{a_{1}}{z-b_{1}-\frac{a_{2}}{z-b_{2}-\cdot}} .}
$$

This relation between the coefficients of the operator $J(t)$ and the rational approximants for its resolvent function gives a procedure for the solution of direct and inverse problems for the operator $J(t)$ with complex coefficients. 
Thus, the direct problem, i.e. the determination of the resolvent function (5) by means of the coefficients $a_{n}(t), b_{n}(t)$ of the operator $J(t)$, can be solved by direct computation of the moments

$$
u_{n}(t)=e_{0}^{T} J^{n}(t) e_{0},
$$

of the resolvent function and then, applying the theory of uniform convergence of the diagonal Padé approximants to the resolvent function (see [9]).

The inverse problem [3], i.e. the determination of the coefficients $a_{n}(t)$, $b_{n}(t)$ of the operator $J(t)$ by means of the resolvent function $S(z ; t)$, can be solved by computing the coefficients of the power series expansion for $S(z ; t)$, and then, applying the algorithm of expansion of a power series (7) for the resolvent function in the continued fraction (9).

Let $\mathcal{P}$ be the column vector of monic orthogonal polynomials with respect to a linear functional $u(t)$ and $J(t)$ the corresponding Jacobi matrix (4). Then, the recurrence relation for the monic orthogonal polynomials can be written as

$$
J(t) \mathcal{P}=x \mathcal{P} .
$$

We shall consider linear functionals normalized to have their first moment equal to one, i.e.

$$
u_{0}(t)=\langle u(t), 1\rangle=1 .
$$

Next, we summarize the known results in relation with the dynamic solutions of the Toda equations (1) and that are dispersed in the literature (see $[3,17,23]$ and references therein):

Theorem 1. Let us assume that the sequences $\left\{a_{n}(t)\right\}_{n \in \mathbb{N}}$ and $\left\{b_{n}(t)\right\}_{n \in \mathbb{N}}$ are uniformly bounded. The following conditions are equivalent:

(1) The Jacobi matrix $J(t)$ defined in (4) satisfies the matrix differential equation $\frac{d}{d t} J(t)=J_{-}(t) J(t)-J(t) J_{-}(t)$, where

$$
J_{-}(t)=\left(\begin{array}{ccccc}
0 & 0 & 0 & & \\
a_{1}(t) & 0 & 0 & 0 & \\
0 & a_{2}(t) & 0 & 0 & 0 \\
& & \ddots & \ddots & \ddots
\end{array}\right)
$$

(2) The moments $u_{n}(t)$, defined by (10), satisfy

$$
\frac{d}{d t} u_{n}(t)=u_{n}(t) u_{1}(t)-u_{n+1}(t), \quad n \in \mathbb{N} .
$$


(3) The Stieltjes or resolvent function associated with $J(t)$ satisfies

$$
\frac{d}{d t} S(z ; t)=-z S(z ; t)+u_{1}(t) S(z ; t)+1 .
$$

(4) The derivative of the linear functional $u(t)$ associated with $J(t)$ satisfies

$$
D u(t)=-x u(t)+u_{1}(t) u(t)
$$

where $x u(t)$ denotes the left product of the linear functional $u(t)$ by the first degree polynomial $x$.

In this work we analyze the correspondence between dynamics of $\Delta$-Toda equations for the coefficients of the Jacobi operator and its resolvent function. Equivalent conditions in terms of difference equation for the Jacobi matrix, the linear functional, the moments and the Stieltjes function are proved. The main ingredient are orthogonal polynomials which satisfy an Appell condition with respect to the forward difference operator $\Delta$. Explicit examples related with Jacobi and Laguerre orthogonal polynomials and $\Delta$-Toda equations are given. As a byproduct relations between confluent hypergeometric series are explicitly given.

\section{2. $\Delta$-Toda equations}

The main aim of this paper is to analyze the following system of difference equations ( $\Delta$-Toda equations):

$$
\left\{\begin{array}{l}
\Delta_{t} a_{n}(t)=\alpha_{1}^{n}(t)\left(b_{n-1}(t)-b_{n}(t+1)\right) \\
\Delta_{t} b_{n}(t)=\alpha_{1}^{n}(t)-\alpha_{1}^{n+1}(t)
\end{array}\right.
$$

where

$$
\alpha_{1}^{n}(t)=\frac{g_{n}(t)}{u_{1}(t+1)+1}
$$

and

$$
g_{n}(t)=\prod_{k=1}^{n} \frac{a_{k}(t+1)}{a_{k-1}(t)},
$$

assuming that $u_{1}(t+1)+1 \neq 0$ and $a_{0}(t)=1$, where the forward difference operator $\Delta_{t}$ is defined by

$$
\Delta_{t} g(t)=g(t+1)-g(t)
$$


We shall also consider the backward difference operator, $\nabla_{t}$, defined by

$$
\nabla_{t} g(t)=g(t)-g(t-1) .
$$

A discrete extension of the results given in Theorem 1 is summarized in the following theorem.

Theorem 2. Let us assume that the sequences $\left\{a_{n}(t)\right\}_{n \in \mathbb{N}}$ and $\left\{b_{n}(t)\right\}_{n \in \mathbb{N}}$ are uniformly bounded. The following conditions are equivalent:

(1) The Jacobi matrix $J(t)$ defined in (4) satisfies the matrix difference equation

$$
\Delta_{t} J(t)=A(t) J(t)-J(t+1) A(t),
$$

where

$$
A(t)=\left(\begin{array}{cccc}
b_{0}(t+1) & 0 & & \\
g_{1}(t) & b_{0}(t+1) & 0 & \\
0 & g_{2}(t) & b_{0}(t+1) & \ddots \\
& \ddots & \ddots & \ddots
\end{array}\right) .
$$

(2) The moments $u_{n}(t)$, defined by (10), satisfy

$$
\Delta_{t} u_{n}(t)=-u_{n+1}(t+1)+u_{1}(t+1) u_{n}(t), \quad n \geq 0 .
$$

(3) The Stieltjes function associated with $J(t)$ satisfies

$$
\Delta_{t} S(z ; t)=-z S(z ; t+1)+u_{1}(t+1) S(z, t)+1 .
$$

(4) The linear functional $u(t)$ associated with $J(t)$ satisfies

$$
\Delta_{t} u(t)=-x u(t+1)+u_{1}(t+1) u(t) .
$$

(5) The monic polynomials $p_{n}(x ; t)$ defined by the three term recurrence relation

$$
p_{n+1}(x ; t)=\left(x-b_{n}(t)\right) p_{n}(x ; t)-a_{n}(t) p_{n-1}(x ; t),
$$

with $p_{-1}(x ; t)=0$ and $p_{0}(x ; t)=1$, satisfy an Appell property

$$
\Delta_{t} p_{n}(x ; t)=\alpha_{1}^{n} p_{n-1}(x ; t),
$$

where

$$
\alpha_{1}^{n}(t)=\frac{\left\langle u(t+1), x^{n} p_{n}(x ; t+1)\right\rangle}{\left(1+u_{1}(t+1)\right)\left\langle u(t), x^{n-1} p_{n-1}(x ; t)\right\rangle}=\frac{g_{n}(t)}{1+u_{1}(t+1)}, n \geq 1 .
$$


Proof: $(1) \Rightarrow(2)$. By induction it can be proved that

$$
\Delta_{t} J^{n}(t)=A(t) J^{n}(t)-J^{n}(t+1) A(t),
$$

where $A(t)$ is defined in (20). By using (10)

$$
e_{0}^{T} \Delta_{t} J^{n}(t) e_{0}=\Delta_{t}\left(e_{0}^{T} J^{n}(t) e_{0}\right)=\Delta_{t} u_{n}(t),
$$

where $e_{0}^{T}=(1,0, \ldots)$. Moreover, from (26) we have

$$
\begin{aligned}
e_{0}^{T} \Delta_{t} J^{n}(t) e_{0}=u_{1}(t & +1) J_{1,1}^{n}(t)-\left(J_{1,1}^{n}(t+1) u_{1}(t+1)\right. \\
& \left.+J_{1,2}^{n}(t+1) a_{1}(t+1)\right)=u_{1}(t+1) u_{n}(t)-J_{1,1}^{n+1}(t+1),
\end{aligned}
$$

since $b_{0}(t+1)=u_{1}(t+1)$, which completes the proof.

$(2) \Rightarrow(3)$. From (7), then

$$
\begin{aligned}
\Delta_{t} S(z ; t) & =\sum_{n=0}^{\infty} \frac{\Delta_{t} u_{n}(t)}{z^{n+1}}=-\sum_{n=0}^{\infty} \frac{u_{n+1}(t+1)}{z^{n+1}}+u_{1}(t+1) \sum_{n=0}^{\infty} \frac{u_{n}(t)}{z^{n+1}} \\
& =-z S(z ; t+1)+u_{1}(t+1) S(z ; t)+1,
\end{aligned}
$$

where we have used that $u_{0}(t)=1$.

$(3) \Rightarrow(4)$. By using

$$
S(z ; t)=\left\langle u(t), \frac{1}{z-x}\right\rangle,
$$

and (11), if we apply the $\Delta_{t}$ operator, we have that the equation (22) reads as

$$
\begin{aligned}
\Delta_{t} S(z ; t):=\left\langle\Delta_{t} u(t), \frac{1}{z-x}\right\rangle & =\left\langle u(t+1), \frac{-z}{z-x}+1\right\rangle+\left\langle u(t), \frac{u_{1}(t+1)}{z-x}\right\rangle \\
& =\left\langle u(t+1), \frac{-x}{z-x}\right\rangle+\left\langle u(t), \frac{u_{1}(t+1)}{z-x}\right\rangle,
\end{aligned}
$$

which implies

$$
\left\langle\Delta_{t} u(t)+x u(t+1)-u_{1}(t+1) u(t), \frac{1}{z-x}\right\rangle=0,
$$

and so, all the moments for the linear functional $\Delta_{t} u(t)+x u(t+1)-u_{1}(t+$ 1) $u(t)$ are zero, and (23) is obtained.

$(4) \Rightarrow(5)$. First of all, let us show that a regular linear functional $u(t)$ satisfying $(21)$, is such that $u_{1}(t+1) \neq-1$. Let us assume that $u_{1}(t+1)=-1$. Then, from $(21)$ we obtain that $u_{2}(t+1)=1$ which yields

$$
\operatorname{det} H_{1}(t+1)=\left|\begin{array}{ll}
u_{0}(t+1) & u_{1}(t+1) \\
u_{1}(t+1) & u_{2}(t+1)
\end{array}\right|=\left|\begin{array}{cc}
1 & -1 \\
-1 & 1
\end{array}\right|=0,
$$


in contradiction with being $u(t)$ a regular linear functional (cf. for instance [5]).

Let $\left\{p_{n}(x ; t)\right\}$ the sequence of monic orthogonal polynomials with respect to the linear functional $u(t)$. Since $\left\{p_{n}(x ; t)\right\}$ is a basis in the space of polynomials of degree $n$, we have

$$
\nabla_{t} p_{n}(x ; t+1)=p_{n}(x ; t+1)-p_{n}(x ; t)=\sum_{k=1}^{n} \alpha_{k}^{n} p_{n-k}(x ; t) .
$$

By convention we shall assume that $\alpha_{1}^{0}=0$. We shall prove for $n>1$ that $\alpha_{k}^{n}=0$ for $k=2, \ldots, n$ and $\alpha_{1}^{n} \neq 0$. By applying the linear functional $u(t)$ to $(27)$ and using the orthogonality of $p_{n}(x ; t)$ it holds

$$
\alpha_{n}^{n}\left\langle u(t), p_{0}(x ; t)\right\rangle=\left\langle u(t), \nabla_{t} p_{n}(x ; t+1)\right\rangle=-\left\langle\Delta_{t} u(t), p_{n}(t+1)\right\rangle,
$$

where we have used that $[1]$

$$
\left\langle\Delta_{t} u, p(x ; t)\right\rangle=-\left\langle u, \nabla_{t} p(x ; t)\right\rangle .
$$

From (23) we obtain

$$
\begin{aligned}
\alpha_{n}^{n}\left\langle u(t), p_{0}(x ; t)\right\rangle & =\left\langle x u(t+1), p_{n}(x ; t+1)\right\rangle-u_{1}(t+1)\left\langle u(t), p_{n}(x ; t+1)\right\rangle \\
& =-u_{1}(t+1)\left\langle u(t), p_{n}(x ; t+1)\right\rangle,
\end{aligned}
$$

for $n>1$, by using the orthogonality. From (27) the above expression can be written as

$$
\begin{aligned}
\alpha_{n}^{n}\left\langle u(t), p_{0}(x ; t)\right\rangle & =-u_{1}(t+1)\left\langle u(t), p_{n}(x ; t)+\sum_{k=1}^{n} \alpha_{k}^{n} p_{n-k}(x ; t)\right\rangle \\
& =-u_{1}(t+1) \alpha_{n}^{n}\left\langle u(t), p_{0}(x ; t)\right\rangle,
\end{aligned}
$$

for $n>1$, by using again the orthogonality. Since $\left\langle u(t), p_{0}(x ; t)\right\rangle=1$,

$$
\left(1+u_{1}(t+1)\right) \alpha_{n}^{n}=0,
$$

and we obtain $\alpha_{n}^{n}=0$. Similar arguments recursively can be used to prove that $\alpha_{k}^{n}=0$, for $k=2, \ldots, n$. Therefore,

$$
\nabla_{t} p_{n}(x ; t+1)=\alpha_{1}^{n} p_{n-1}(x ; t) .
$$

Finally, we will determine $\alpha_{1}^{n}$ by using the orthogonality of $p_{n}(x ; t)$ :

$$
\begin{aligned}
\alpha_{1}^{n}\left\langle u(t), x^{n-1} p_{n-1}(x ; t)\right\rangle=\left\langle u(t), \nabla_{t}\left(x^{n-1} p_{n}(x ; t+1)\right)\right\rangle \\
=-\left\langle\Delta_{t} u(t), x^{n-1} p_{n}(x ; t+1)\right\rangle .
\end{aligned}
$$


In order to compute the last inner product we shall use

$$
p_{n}(x ; t+1)=p_{n}(x ; t)+\alpha_{1}^{n} p_{n-1}(x ; t),
$$

as well as the orthogonality:

$$
\begin{aligned}
& \left\langle\Delta_{t} u(t), x^{n-1} p_{n}(x ; t+1)\right\rangle \\
& \quad=\left\langle u(t+1), x^{n} p_{n}(x ; t+1)\right\rangle-u_{1}(t+1)\left\langle u(t), x^{n-1} p_{n}(x ; t+1)\right\rangle \\
& \quad=\left\langle u(t+1), x^{n} p_{n}(x ; t+1)\right\rangle-u_{1}(t+1)\left\langle u(t), x^{n-1}\left(p_{n}(x ; t)+\alpha_{1}^{n} p_{n-1}(x ; t)\right)\right\rangle \\
& \quad=\left\langle u(t+1), x^{n} p_{n}(x ; t+1)\right\rangle-u_{1}(t+1) \alpha_{1}^{n}\left\langle u(t), x^{n-1} p_{n-1}(x ; t)\right\rangle
\end{aligned}
$$

which gives the value of $\alpha_{1}^{n}$ given in (25).

$(5) \Rightarrow(1)$ If we apply $\nabla_{t}$ to the recurrence relation

$x p_{n}(x ; t+1)=p_{n+1}(x ; t+1)+b_{n}(t+1) p_{n}(x ; t+1)+a_{n}(t+1) p_{n-1}(x ; t+1)$,

we get

$$
\begin{aligned}
& \alpha_{1}^{n} x p_{n-1}(x ; t)=\alpha_{1}^{n+1} p_{n}(t)+b_{n}(t+1) \alpha_{1}^{n} p_{n-1}(x ; t) \\
& \quad+\Delta_{t} b_{n}(t) p_{n}(x ; t)+a_{n}(t+1) \alpha_{1}^{n-1} p_{n-2}(x ; t)+\Delta_{t} a_{n}(t) p_{n-2}(x ; t) .
\end{aligned}
$$

If we use again the recurrence relation to expand

$$
x p_{n-1}(x ; t)=p_{n}(x ; t)+b_{n-1}(t) p_{n-1}(x ; t)+a_{n-1}(t) p_{n-2}(x ; t),
$$

by equating the coefficients in $p_{n}(x, t), p_{n-1}(x ; t)$ and $p_{n-2}(x ; t)$, we get the $\Delta$-Toda equations

$$
\left\{\begin{array}{l}
\alpha_{1}^{n}(t)=\alpha_{1}^{n+1}(t)+\Delta_{t} b_{n}(t), \\
\alpha_{1}^{n}(t)\left(b_{n-1}(t)-b_{n}(t+1)\right)=\Delta_{t} a_{n}(t),
\end{array}\right.
$$

with $\alpha_{1}^{n}(t) a_{n-1}(t)=\alpha_{1}^{n-1}(t) a_{n}(t+1), n \in \mathbb{N}$, which completes the proof.

Theorem 3. In the hypothesis of Theorem 2, assume that the normalized functional $u(t)$ verifies

$$
u(t)=\kappa(1+x)^{1-t} v,
$$

where $\kappa$ is the normalizing constant and $v$ is a positive definite linear functional. Then, the coefficients $\left\{a_{n}(t)\right\}_{n \in \mathbb{N}},\left\{b_{n}(t)\right\}_{n \in \mathbb{N}}$ of the Jacobi matrix $J(t)$ associated to $u(t)$ are solution of the $\Delta$-Toda equations (16). 
Proof: Let

$$
f(x, t)=(1+x)^{1-t}
$$

and the moments

$$
\left\langle v, x^{n}\right\rangle=\int x^{n} d \varrho(x), \quad n=0,1, \ldots
$$

Let $u_{n}(t)$ the moments of the linear functional $u(t)$,

$$
u_{n}(t)=\frac{\int f(x, t) x^{n} d \varrho(x)}{\int f(x, t) d \varrho(x)} .
$$

Since

then

$$
\Delta_{t}(f(t) / g(t))=\frac{\Delta f(t) g(t)-f(t) \Delta g(t)}{g(t) g(t+1)}
$$

$$
\Delta_{t} u_{n}(t)=\frac{\int \Delta_{t} f(x, t) x^{n} d \varrho(x)}{\int f(x, t+1) d \varrho(x)}-\frac{\left(\int f(x, t) x^{n} d \varrho(x)\right)\left(\int \Delta_{t} f(x, t) d \varrho(x)\right)}{\left(\int f(x, t) d \varrho(x)\right)\left(\int f(x, t+1) d \varrho(x)\right)} .
$$

By using $\Delta_{t} f(x, t)=-x f(x, t+1)$, we obtain

$$
\Delta_{t} u_{n}(t)=-u_{n+1}(t+1)+u_{1}(t+1) u_{n}(t),
$$

which completes the proof.

Remark 1. Let us consider the difference operator

$$
\Delta_{t, h} f(x, t)=\frac{f(x, t+h)-f(x, t)}{h}, \quad \lim _{h \rightarrow 0} \Delta_{t, h} f(x, t)=\frac{\partial}{\partial t} f(x, t) .
$$

In this case, the function $f_{h}(x, t)$ to be considered analogue of $(30)$ is

$$
f_{h}(x, t)=\left(\frac{1}{1+h / x}\right)^{-1+t / h} \text {. }
$$

It yields,

$$
\lim _{h \rightarrow 0}\left(\frac{1}{1+h / x}\right)^{-1+t / h}=\exp (-x t),
$$

which is the evolution (3) associated to the continuous case $[12,19]$.

Next, we prove a Lax-type theorem [15, Theorem 3, p. 270].

Theorem 4. In the hypothesis of Theorem 2, let $\lambda(t)$ be a spectral point of $J(t)$ such that verifies (19). Then,

$$
\Delta_{t} \lambda(t)=0 .
$$


Proof: If we apply the $\Delta_{t}$ operator to

$$
J(t) \mathcal{P}(\lambda(t))=\lambda(t) \mathcal{P}(\lambda(t))
$$

we obtain

$$
\Delta_{t} J(t) \mathcal{P}(\lambda(t))+J(t+1) \Delta_{t} \mathcal{P}(\lambda(t))=\Delta_{t} \lambda(t) \mathcal{P}(\lambda)+\lambda(t+1) \Delta \mathcal{P}(\lambda(t)) .
$$

Then,

$$
\begin{array}{r}
A \lambda(t) \mathcal{P}(\lambda(t))-J(t+1) A \mathcal{P}(\lambda(t))+(J(t+1)-\lambda(t+1) \mathcal{I}) \Delta_{t} \mathcal{P}(\lambda(t)) \\
=\left(\Delta_{t} \lambda(t)\right) \mathcal{P}(\lambda(t)) .
\end{array}
$$

Since $(J(t+1)-\lambda(t+1) \mathcal{I})\left(\Delta_{t} \mathcal{P}(\lambda(t))-A \mathcal{P}(\lambda(t))\right)=0$, it yields

$$
\left(\Delta_{t} \lambda(t) \mathcal{I}+(\lambda(t+1)-\lambda(t)) A\right) \mathcal{P}(\lambda(t))=0 .
$$

The element $(1,1)$ of the above column vector is given by

$$
(\lambda(t)-\lambda(t+1)) u_{1}(t+1)=\Delta_{t} \lambda(t)
$$

and then

$$
\left(1+u_{1}(t+1)\right) \Delta_{t} \lambda(t)=0
$$

which gives the result by using that $u_{1}(t+1)+1 \neq 0$.

\section{Examples}

3.1. Modified Jacobi functional. Let $v^{(\alpha, \beta)}$ be the normalized Jacobi linear functional, with $\alpha, \beta>-1$, defined as

$$
\left\langle v^{(\alpha, \beta)}, p(x)\right\rangle=\int_{-1}^{1} p(x)(1-x)^{\alpha}(1+x)^{\beta} \frac{2^{-\alpha-\beta-1} \Gamma(\alpha+\beta+2)}{\Gamma(\alpha+1) \Gamma(\beta+1)} d x .
$$

We have that

$$
\left\langle v^{(\alpha, \beta)}, 1\right\rangle=1, \quad\left\langle v^{(\alpha, \beta)}, x\right\rangle=\frac{\beta-\alpha}{\alpha+\beta+2} .
$$

Let us consider — see (29) -

$$
u(t)=\kappa(1+x)^{1-t} v^{(\alpha, \beta)},
$$

where $\kappa$ is a normalizing constant. In terms of measures the linear functional $u(t)$ has associated the weight function

$$
\varrho(x, t ; \alpha, \beta)=\frac{2^{-\alpha-\beta+t-2} \Gamma(\alpha+\beta-t+3)}{\Gamma(\alpha+1) \Gamma(\beta-t+2)}(1-x)^{\alpha}(1+x)^{\beta-t+1},
$$


where $\alpha>-1$ and $\beta>t-2$. We have that

$$
\int_{-1}^{1} \varrho(x, t ; \alpha, \beta)=1
$$

and moreover from (31) it yields

$$
u_{1}(t)=\int_{-1}^{1} x \varrho(x, t ; \alpha, \beta)=\frac{-\alpha+\beta-t+1}{\alpha+\beta-t+3} .
$$

Thus,

$$
u_{1}(t+1)=\frac{-\alpha+\beta-t}{\alpha+\beta-t+2}=1-\frac{2(\alpha+1)}{\alpha+\beta-t+2} \neq-1 .
$$

As it is well-known, monic Jacobi polynomials $P_{n}^{(\alpha, \beta)}(x)$ satisfy the following three term recurrence relation

$$
P_{n+1}^{(\alpha, \beta)}(x)=\left(x-B_{n}^{(\alpha, \beta)}\right) P_{n}^{(\alpha, \beta)}(x)-A_{n}^{(\alpha, \beta)} P_{n-1}^{(\alpha, \beta)}(x),
$$

with initial conditions $P_{-1}^{(\alpha, \beta)}(x)=0, P_{0}^{(\alpha, \beta)}(x)=1$, where

$$
\begin{aligned}
B_{n}^{(\alpha, \beta)} & =\frac{\beta^{2}-\alpha^{2}}{(\alpha+\beta+2 n)(\alpha+\beta+2 n+2)}, \\
A_{n}^{(\alpha, \beta)} & =\frac{4 n(\alpha+n)(\beta+n)(\alpha+\beta+n)}{(\alpha+\beta+2 n-1)(\alpha+\beta+2 n)^{2}(\alpha+\beta+2 n+1)} .
\end{aligned}
$$

Thus, the polynomials orthogonal with respect to $u(t)$ defined in (32) satisfy a three term recurrence relation as above with coefficients $b_{n}(t)=B_{n}^{(\alpha, \beta-t+1)}$ and $a_{n}(t)=A_{n}^{(\alpha, \beta-t+1)}$.

From (18), we have

$$
\begin{aligned}
g_{n}(t) & =\prod_{k=1}^{n} \frac{A_{k}^{(\alpha, \beta-(t+1)-1)}}{A_{k-1}^{(\alpha, \beta-t+1)}} \\
& =\frac{4 n(\alpha+n)(\beta-t+1)}{(\alpha+\beta-t+2)(\alpha+\beta+2 n-t)(\alpha+\beta+2 n-t+1)},
\end{aligned}
$$

and therefore

$$
\alpha_{1}^{n}(t)=\frac{g_{n}(t)}{u_{1}(t+1)+1}=\frac{2 n(\alpha+n)}{(\alpha+\beta+2 n-t)(\alpha+\beta+2 n-t+1)} .
$$

Thus, we have that $b_{n}(t)=B_{n}^{(\alpha, \beta-t+1)}, a_{n}(t)=A_{n}^{(\alpha, \beta-t+1)}$ and $\alpha_{1}^{n}(t)$ are solution of the $\Delta$-Toda equations (16). 
3.2. Modified Laguerre functional. Let us consider the Laguerre linear functional, with $\alpha>-1$ defined as

$$
\left\langle v^{(\alpha)}, p(x)\right\rangle=\int_{0}^{\infty} p(x) \frac{\exp (-x) x^{\alpha}}{\Gamma(\alpha+1)} d x .
$$

We have that the moments are explicitly given by

$$
\left\langle v^{(\alpha)}, x^{n}\right\rangle=\frac{\Gamma(\alpha+n+1)}{\Gamma(\alpha+1)}=(\alpha+1)_{n},
$$

where $(A)_{n}=A(A+1) \cdots(A+n-1)$ with $(A)_{0}=1$ denotes the Pochhammer symbol.

Let us consider

$$
u(t)=\kappa(1+x)^{1-t} v^{(\alpha)},
$$

where $\kappa$ is a normalizing constant. In terms of measures, the linear functional $u(t)$ has associated the weight

$$
\varrho(x ; t ; \alpha)=(1+x)^{1-t} \frac{\exp (-x) x^{\alpha}}{U(1+\alpha, 3+\alpha-t, 1) \Gamma(\alpha+1)}, \quad x \geq 0,
$$

where

$$
U(a, b, z)=\frac{1}{\Gamma(a)} \int_{0}^{\infty} e^{-z t} t^{a-1}(1+t)^{b-a-1} d t
$$

is the confluent hypergeometric function [21, 22].

We have that

$$
\int_{0}^{\infty} \varrho(x ; t ; \alpha)=1
$$

and moreover the moments of $u(t)$ can be written as

$$
\begin{aligned}
u_{n}(t)=\left\langle u(t), x^{n}\right\rangle & =\int_{0}^{\infty} x^{n} \varrho(x ; t ; \alpha) \\
& =\frac{\Gamma(\alpha+n+1) U(t-1,-\alpha-n+t-1,1)}{\Gamma(\alpha+1) U(t-1,-\alpha+t-1,1)} .
\end{aligned}
$$

In particular

$$
u_{1}(t+1)=\frac{(\alpha+1) U(t,-\alpha+t-1,1)}{U(t, t-\alpha, 1)} \neq-1 .
$$


The moments $u_{n}(t)$ satisfy relation (21) which in this case provides the following relation involving confluent hypergeometric functions

$$
\begin{aligned}
& \frac{U(t,-\alpha-n+t, 1)}{U(t, t-\alpha, 1)}-\frac{U(\alpha+n+1, \alpha+n-t+3,1)}{U(\alpha+1, \alpha-t+3,1)} \\
& =-\frac{(\alpha+n+1) U(\alpha+n+2, \alpha+n-t+3,1)}{U(t, t-\alpha, 1)} \\
& \quad+\frac{(\alpha+1) U(t,-\alpha+t-1,1) U(\alpha+n+1, \alpha+n-t+3,1)}{U(\alpha+1, \alpha-t+3,1) U(t, t-\alpha, 1)} .
\end{aligned}
$$

\section{References}

[1] F. Abdelkarim and P. Maroni. The $D_{\omega}$-classical orthogonal polynomials. Results Math., 32(12):1-28, 1997.

[2] M. J. Ablowitz and H. Segur. Solitons and the inverse scattering transform, volume 4 of SIAM Studies in Applied Mathematics. Society for Industrial and Applied Mathematics (SIAM), Philadelphia, Pa., 1981.

[3] A. I. Aptekarev and A. Branquinho. Padé approximants and complex high order Toda lattices. Journal of Computational and Applied Mathematics, 155(2):231 - 237, 2003.

[4] A. I. Aptekarev, V. Kaliaguine, and W. Van Assche. Criterion for the resolvent set of nonsymmetric tridiagonal operators. Proc. Amer. Math. Soc., 123(8):2423-2430, 1995.

[5] T. S. Chihara. An introduction to orthogonal polynomials. Gordon and Breach Science Publishers, New York, 1978.

[6] C. S. Gardner, J. M. Greene, M. D. Kruskal, and R. M. Miura. Method for solving the Korteweg-de Vries equation. Phys. Rev. Lett., 19:1095-1097, Nov 1967.

[7] C. S. Gardner, J. M. Greene, M. D. Kruskal, and R. M. Miura. Korteweg-deVries equation and generalization. VI. Methods for exact solution. Comm. Pure Appl. Math., 27:97-133, 1974.

[8] J. Gilewicz. 100 years of improvements of bounding properties of Padé approximants to the Stieltjes functions: one-point, two-point and $N$-point Padé approximants. Appl. Numer. Math., 60(12):1320-1331, 2010.

[9] A. A. Gonchar. Uniform convergence of diagonal Padé approximants. Mat. Sb. (N.S.), 118(160)(4):535-556, 1982.

[10] B. Grammaticos and A. Ramani. Discrete Painlevé equations: A review. In Basil Grammaticos, Thamizharasi Tamizhmani, and Yvette Kosmann-Schwarzbach, editors, Discrete Integrable Systems, volume 644 of Lecture Notes in Physics, pages 245-321. Springer Berlin Heidelberg, 2004.

[11] A. Its. Symmetries and Integrability of Difference Equations, volume 301 of London Mathematical Society Lecture Note Series, Chapter 5: Discrete Painlevé Equations and Orthogonal Polynomials, pages 139-159. Cambridge University Press, 2011.

[12] M. Kac and P. van Moerbeke. On an explicitly soluble system of nonlinear differential equations related to certain Toda lattices. Adv. Math., 16(2):160-169, 1975.

[13] D. J. Korteweg and G. de Vries. XLI. On the change of form of long waves advancing in a rectangular canal, and on a new type of long stationary waves. Philosophical Magazine Series 5, 39(240):422-443, 1895.

[14] P. D. Lax. Integrals of non-linear equations of evolution and solitary waves, Comm. Pure Appl. Math., 21 (1968), 467-490. 
[15] P. D. Lax. Linear algebra and its applications. Pure and Applied Mathematics (Hoboken). Wiley-Interscience [John Wiley \& Sons], Hoboken, NJ, second edition, 2007.

[16] R. S. Leite and C. Tomei. Parameterization by polytopes of intersections of orbits by conjugation. Linear Algebra Appl., 361:223-243, 2003.

[17] A. P. Magnus. Painlevé equations for semiclassical recurrence coefficients: Research problems 96-2. Constr. Approx., 12(2):303-306, 1996.

[18] P. Maroni. Prolégomènes à l'étude des polynômes orthogonaux semi-classiques. Ann. Mat. Pura Appl. (4), 149:165-184, 1987.

[19] J. Moser. Three integrable Hamiltonian systems connected with isospectral deformations. Adv. Math., 16:197-220, 1975.

[20] E. M. Nikishin and V. N. Sorokin. Rational approximations and orthogonality, volume 92 of Translations of Mathematical Monographs. American Mathematical Society, Providence, RI, 1991.

[21] NIST Digital Library of Mathematical Functions. http://dlmf.nist.gov/, Release 1.0.8 of 201404-25. Online companion to [22].

[22] F. W. J. Olver, D. W. Lozier, R. F. Boisvert, and C. W. Clark, editors. NIST Handbook of Mathematical Functions. Cambridge University Press, New York, NY, 2010. Print companion to $[21]$.

[23] F. Peherstorfer. On Toda lattices and orthogonal polynomials. J. Comput. Appl. Math. 133:519-534, 2001.

[24] A. M. Perelomov. Integrable systems of classical mechanics and Lie algebras. Vol. I. Birkhäuser Verlag, Basel, 1990.

[25] S. N. M. Ruijsenaars. Relativistic Toda systems. Comm. Math. Phys., 133(2):217-247, 1990.

[26] G. Szegö, Orthogonal Polynomials, Amer. Math. Soc. Colloq. Publ., vol. 23, Amer. Math. Soc., Providence Rhode Island, 1975 (fourth edition).

[27] M. Toda. Vibration of a chain with nonlinear interaction. Journal of the Physical Society of Japan, 22(2):431-436, 1967.

\section{AREA}

Departamento de Matemática Aplicada iI, E.E. Telecomunicación, Universidade de Vigo, 36310-Vigo, SPAin.

E-mail address: area@uvigo.es

A. Branquinho CMUC and Department of Mathematics, University of Coimbra, Apartado 3008, EC Santa Cruz, 3001-501 COIMBRA, Portugal.

E-mail address: ajplb@mat.uc.pt

A. Foulquié Moreno Cidma, Department of Mathematics, University of Aveiro, Campus de Santiago, 3810193 Aveiro, Portugal.

E-mail address: foulquie@ua.pt

E. GODOY

Departamento de Matemática Aplicada II, E.E. Industrial, Universidade de Vigo, Campus Lagoas-Marcosende, 36310 Vigo, Spain.

E-mail address: egodoy@dma.uvigo.es 